\title{
A ligand for peroxisome proliferator activated receptor $\gamma$ inhibits cell growth and induces apoptosis in human liver cancer cells
}

\author{
M Toyoda, H Takagi, N Horiguchi, S Kakizaki, K Sato, H Takayama, M Mori
}

See end of article for authors' affiliations

\section{Correspondence to:} Dr H Takagi, First Department of Internal Medicine, Gunma University School of Medicine, 3-39-15 Showa-machi, Maebashi, Gunma 371-8511, Japan; htakagi@med.gunma-u.ac.jp

Accepted for publication 5 June 2001

\begin{abstract}
Background and aims: Induction of apoptosis of cancer cells through ligands of nuclear hormone receptors (NHRs) is a new approach in cancer therapy. Recently, one of the NHRs, peroxisome proliferator activated receptor $\gamma$ (PPAR $\gamma$ ), has been shown to influence cell growth in certain cancer cells although its effect on hepatocellular carcinoma $(\mathrm{HCC})$ has not been analysed.

Methods: Experiments were conducted using three human liver cancer cell lines, PLC/PRF/5, Hep G2 and $\mathrm{HuH}-7$, in vitro. These cells were exposed to troglitazone, a synthetic ligand for PPAR $\gamma$, and the effects on cell growth were analysed.

Results: Expression of PPAR $\gamma$ mRNA was detected in all three liver cancer cell lines. Activation of PPAR $\gamma$ by troglitazone caused a marked growth inhibition in a dose dependent manner in three hepatoma cell lines. The DNA fragmentation ELISA assay and Hoechst 33258 staining revealed that the growth inhibitory effect by adding troglitazone was due to apoptosis of $\mathrm{PLC} / \mathrm{PRF} / 5$, which strongly expressed PPAR $\gamma$. Troglitazone also induced activation of the cell death protease, caspase 3, but not caspase 8, in PLC/PRF/5 cells. However, expression levels of antiapoptotic factor bcl-2 and apoptosis inducing factor bax were not affected.

Conclusion: Our study showed that PPAR $\gamma$ was expressed in human liver cancer cells and that the ligand for PPAR $\gamma$, troglitazone, inhibited the growth of these cells by inducing apoptosis through caspase 3 activation, indicating that troglitazone could be potentially useful as an apoptosis inducer for the treatment of HCC.
\end{abstract}

$\mathrm{H}$ epatocellular carcinoma (HCC) is one of the most prevalent cancers in the world. ${ }^{12}$ Because of the clinical characteristics of HCC, such as association with chronic liver disease and multicentric hepatocarcinogenesis, an effective treatment is not fully established and the prognosis is still poor. Because of the persistent rise in the prevalence of HCC and limited therapeutic options, there is an immediate need for identifying a new molecular target for anticancer therapy in HCC. As one of the evolving cancer therapies, induction of apoptosis through ligands of nuclear hormone receptors (NHRs) has been previously reported. ${ }^{34}$

Peroxisome proliferator activated receptor $\gamma$ (PPAR $\gamma)$ is a member of the NHR super family, which includes receptors for steroids, thyroid hormone, and retinoic acid. ${ }^{5}$ PPAR $\gamma$ is highly expressed in adipose tissues and is an important regulator of adipocyte differentiation. Activation of this receptor in cultured preadipocytes causes lipid accumulation and activation of several genes characteristic of adipocytes. ${ }^{6}$ The synthetic drug troglitazone has been known as a relatively high affinity ligand for PPAR $\gamma$ and was used widely in patients with insulin resistant diabetes mellitus. Recent data showed that ligands for PPAR $\gamma$ exhibit growth inhibitory effects on colon cancer, breast cancer, prostate cancer, and gastric cancer by affecting differentiation and/or inducing apoptosis ${ }^{7-10}$ both in vitro and in vivo. Since some liver cancer cells, especially in the well differentiated type, demonstrated accumulation of fatty droplets, transformation of hepatocytes into HCC may be associated with dysregulation of lipid metabolism. In this study, we analysed the possible role of PPAR $\gamma$ and the effect of troglitazone on liver cancer cells to examine its therapeutic effect in HCC.

\section{MATERIALS AND METHODS}

Analysis of cell growth

Human liver cancer cell lines PLC/PRF/5, Hep G2, and HuH-7 were cultured in Dulbecco's modified Eagle's medium (Gibco
BRL, Grand Island, New York, USA) containing $10 \%$ fetal bovine serum (Equitech-Bio, Ingram, Texas, USA), $50 \mathrm{U} / \mathrm{ml}$ penicillin, and $50 \mu \mathrm{g} / \mathrm{ml}$ streptomycin (Gibco BRL). Cells were incubated at $37^{\circ} \mathrm{C}$ in a humidified chamber of $5 \% \mathrm{CO}_{2}$.

The synthetic ligand for PPAR $\gamma$, troglitazone, was kindly provided by Sankyo Pharmaceuticals (Tokyo, Japan). Troglitazone was dissolved at appropriate concentrations in dimethyl sulphoxide (Wako, Tokyo, Japan) and in cell culture medium at a final concentration of less than $0.1 \%(\mathrm{vol} / \mathrm{vol})$.

HCC cell lines were seeded in 96 well microtitre plates (Nunc Inc., Napervill, Illinois, USA) at a cell density of $2 \times 10^{4}$ cells $/ 0.1 \mathrm{ml} /$ well for PLC/PRF/5 and HuH-7, and at $4 \times 10^{4}$ cells/ $0.1 \mathrm{ml} /$ well for Hep G2. After overnight incubation, the medium was replaced with $0.1 \mathrm{ml}$ of fresh medium containing different concentrations of troglitazone and microtitre plates were incubated for $24,48,72$, and 120 hours. Cell growth was measured by MTT (3-(4,5-dimethylthiazol-2-yl)-2,5-diphenyl tetrazolium bromide) assay (Sigma, St Louis, Missouri, USA). The number of viable cells was estimated by measuring absorbance (OD 550 values) in a microplate reader at $550 \mathrm{~nm}$ with a reference wavelength of $650 \mathrm{~nm}$.

\section{RNA analysis}

Total cellular RNA was extracted using Isogen (Nippon Gene, Toyama, Japan) according to the protocol recommended by the manufacturer. In this assay, $20 \mu \mathrm{g}$ of total RNA sample was fractionated by electrophoresis through $1 \%$ agarose gel and then blotted onto Hybond-N nylon membranes (Amersham

Abbreviations: NHR, nuclear hormone receptor; PPAR $\gamma$, peroxisome proliferator activated receptor $\gamma ; \mathrm{HCC}$, hepatocellular carcinoma; $N F \kappa B$, nuclear factor kappa B; Ab, antibody. 
Life Science, Buckinghamshire, UK), followed by ultraviolet cross linking. Hybridisation was carried out at $42^{\circ} \mathrm{C}$ for 12 hours with ${ }^{32} \mathrm{P}$ labelled cDNA probes. The probe for human PPAR $\gamma$ was kindly provided by Dr Spiegelmann. ${ }^{6}$

\section{Quantification of DNA fragmentation by ELISA}

DNA fragmentation was measured using a cellular DNA fragmentation ELISA kit (Roche Diagnosis, Mannheim, Germany), as described in the protocol provided by the manufacturer. Briefly, liver cancer cell line PLC/PRF/5 was adjusted to $1 \times 10^{4}$ cells/well in a microtitre plate. After overnight BrdU labelling, cells were treated with $0,5,10$, and $100 \mu \mathrm{M}$ of troglitazone for 72 hours. Cells were lysed and the microtitre plate was centrifuged at $250 \mathrm{~g}$ for 10 minutes. Then, $100 \mu \mathrm{l}$ of the supernatant were removed and analysed by ELISA, using the instructions recommended by the manufacturer.

\section{Detection of apoptotic cells}

Nuclear morphology was assessed using Hoechst staining. PLC/PRF/5 was incubated with $20 \mu \mathrm{M}$ of troglitazone for 72 hours. Cells were washed with Dulbecco's modified Eagle's medium and labelled with Hoechst 33258 (Sigma) for 10 minutes. The cells were examined by fluorescence microscopy. Apoptotic cells were distinguished by their characteristic patterns of nuclear condensation, cytoplasmic rounding.

\section{Detection of caspase activity}

$\mathrm{PLC} / \mathrm{PRF} / 5$ was treated with troglitazone for 48 hours and activation of caspase 3 and caspase 8 was analysed using 250 $\mu g$ of cell lysate by the colorimetric protease assay kit (MBL, Aichi, Japan), as recommended by the manufacturer. The activity of caspase was converted through measurement of absorbance (OD 405 values) using a microplate reader at 405 nm. Non-specific reaction was corrected by subtracting the background readings from the combination of cell lysates and buffers.

\section{Western blot analysis}

To determine protein expression of bcl-2 and bax, western blot analysis was performed. PLC/PRF/5, treated with various concentrations of troglitazone for 96 hours, was lysed in lysis buffer ( $10 \mathrm{mM}$ Tris $\mathrm{HCl}, \mathrm{pH} 7.4,150 \mathrm{mM} \mathrm{NaCl}, 1 \%$ Nonidet P-40, $1 \mathrm{mM}$ EDTA, $0.1 \%$ sodium dodecyl sulphate, $0.1 \%$ deoxycholic acid, and protease inhibitor mixture). Following centrifugation at $14000 \mathrm{rpm}$ at $4^{\circ} \mathrm{C}$ for 15 minutes, the protein concentration of the supernatants was determined using the Bio-Rad protein assay kit (Bio-Rad, Hercules, California, USA). A total of $30 \mu \mathrm{g}$ of protein per lane was run on sodium dodecyl sulphate-polyacrylamide gel electrophoresis and then transferred onto nitrocellulose membranes for western blotting (Trans-Blot Transfer Medium; Bio-Rad). Membranes were blocked overnight at $4{ }^{\circ} \mathrm{C}$ in a milk blocking buffer $(5 \%$ non-fat dry milk). The blots were then probed for two hours with primary antibody $(\mathrm{Ab})$ diluted in blocking buffer $(20 \mathrm{mM}$ Tris $\mathrm{HCl}, \mathrm{pH} 7.4,150 \mathrm{mM} \mathrm{NaCl}$ solution containing $0.1 \%$ Tween 20). Rabbit anti-bcl-2 polyclonal Ab (Calbiochem, San Diego, California, USA; diluted $1 / 500$ ), mouse anti-bax monoclonal Ab (Santa Cruz Biotechnology, Santa Cruz, California, USA; diluted $1 / 100)$, and mouse anti- $\beta$-actin monoclonal $\mathrm{Ab}$ (Sigma; diluted 1/2000) were used. Following five washes, the blots were incubated for one hour with horseradish peroxidase conjugated secondary Ab (Toyobo, Osaka, Japan; diluted 1/2000). After additional washing, membranes were developed by western blotting luminol reagent from Santa Cruz Biotechnology.

\section{Statistical analysis}

Results are expressed as mean (SD). Statistical analysis was performed by repeated measures analysis of variance followed by Fisher's PLSD test. A value of $p<0.05$ was considered statistically significant.

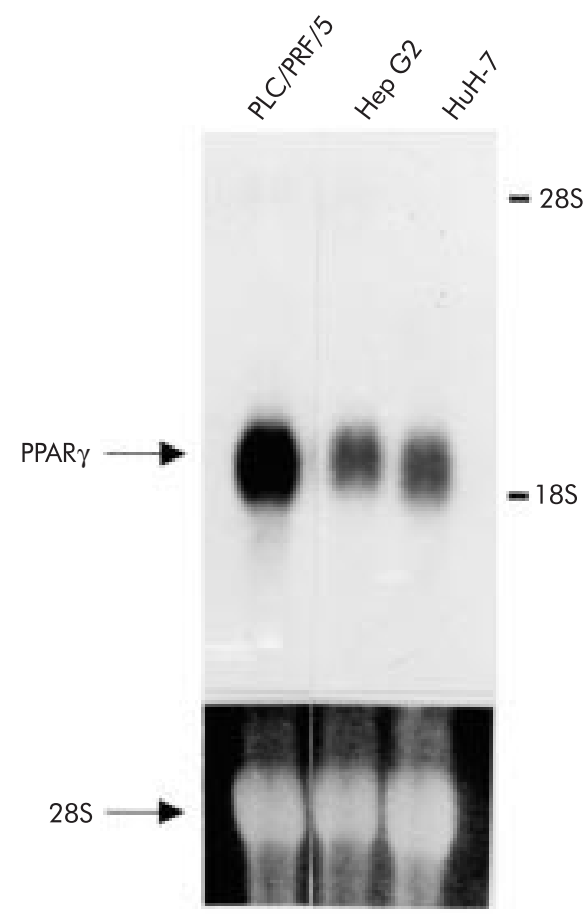

Figure 1 Northern blot analysis revealed peroxisome proliferator activated receptor $\gamma$ (PPAR $\gamma$ ) expression in all three liver cancer cell lines (PLC/PRF/5, Hep G2, and HuH-7). The expression level of PPAR $\gamma$ mRNA was higher in PLC/PRF/5 than in Hep G2 or HuH-7.

\section{RESULTS}

\section{Expression of PPAR $\gamma$ in human liver cell lines}

Although recent data showed that several cell lines of colon cancer, breast cancer, prostate cancer, and gastric cancer expressed PPAR $\gamma$ mRNA, ${ }^{7-10}$ expression of PPAR $\gamma$ mRNA in liver cancer cell lines has not been studied. Expression of PPAR $\gamma$ mRNA was detected by northern blot analysis in all three liver cancer cell lines used in the present study (fig l). The expression level of PPAR $\gamma$ was higher in PLC/PRF/5 than in the two other cell lines (Hep G2 and HuH-7).

Troglitazone inhibits growth of liver cancer cells in vitro Three liver cancer cell lines were continuously cultured in the presence of various concentrations of troglitazone to investigate the effect of troglitazone on cell growth. Significantly, troglitazone markedly inhibited cell growth in all three cell lines. The growth inhibitory effect of troglitazone on $\mathrm{PLC} / \mathrm{PRF} / 5$ was similar to that on $\mathrm{HuH}-7$ with an effective dose $\left(\mathrm{EC}_{5_{0}}\right.$ ) of $10 \mu \mathrm{M}$ (fig $2 \mathrm{~A}$ ). In contrast, Hep G2 was less sensitive to the inhibitory effect of troglitazone than Huh-7 and PLC/PRF/5 (120 hours). Treatment with $10 \mu \mathrm{M}$ of troglitazone resulted in significant growth inhibition in all three cell lines in time course studies over 120 hours (fig 2B).

\section{Troglitazone induces cell apoptosis}

As the growth inhibitory effect of troglitazone is reported to be due in part to induction of apoptosis, ${ }^{11}$ we carried out the following experiments. Culture of PLC/PRF/5 for 72 hours in the presence of 10,50 , or $100 \mu \mathrm{M}$ of troglitazone was associated with cellular DNA fragmentation measured by ELISA, which increased proportionately with increasing concentrations of troglitazone (fig 3). We also evaluated chromatin morphology using Hoechst 33258 staining (fig 4). PLC/PRF/5 treated with $20 \mu \mathrm{M}$ of troglitazone for 72 hours showed morphological features of apoptosis. In contrast, untreated cells maintained normal chromatin patterns and cell size. The number of apoptotic cells was proportional to the dose of troglitazone (data not shown). 

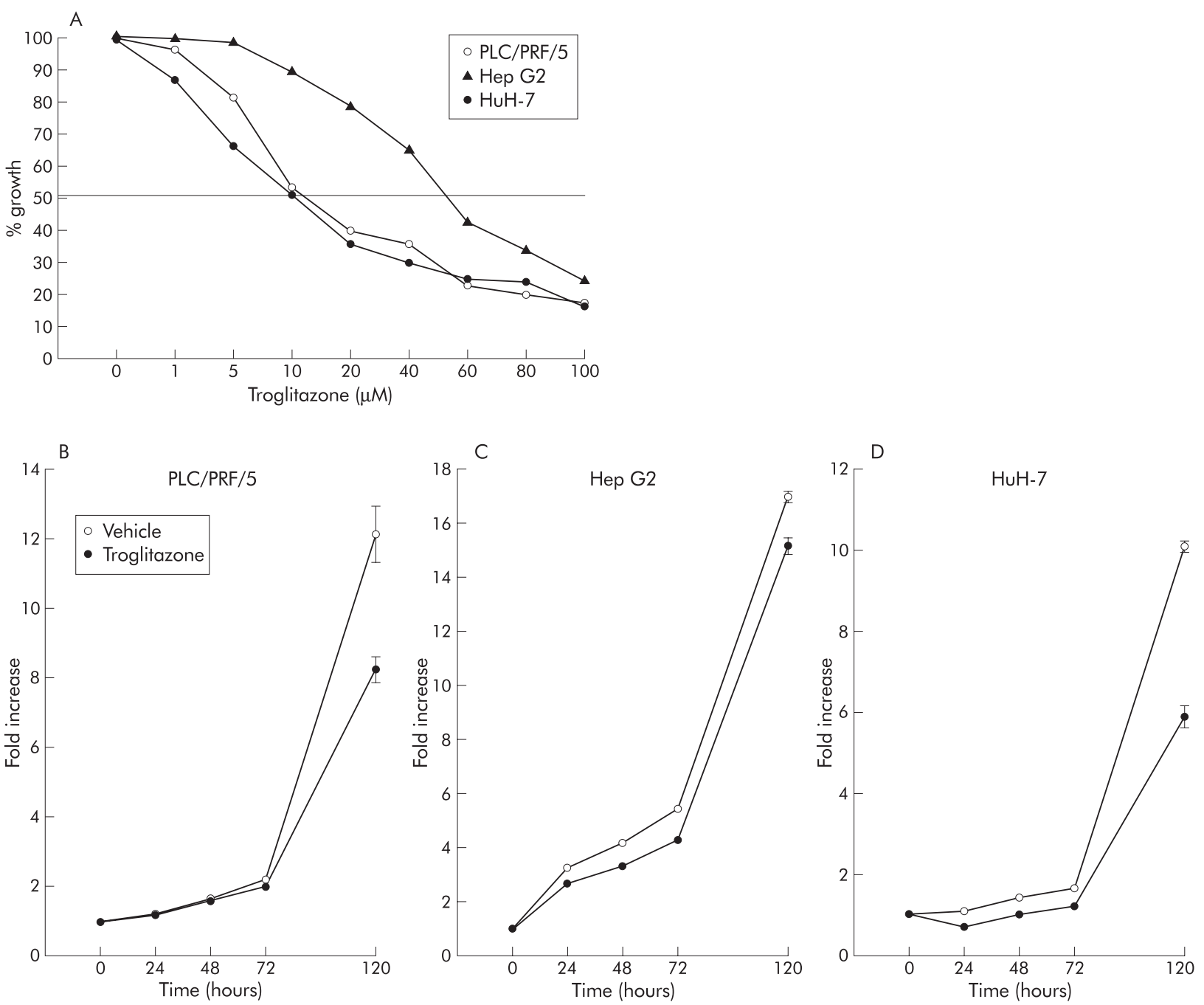

Figure 2 (A) Effect of troglitazone on the growth of liver cancer cell lines. The growth of all three liver cancer cell lines was inhibited by troglitazone treatment in a dose dependent manner. Data are presented as per cent growth relative to untreated cells (120 hours). (B-D) Time course of inhibition of cell growth by troglitazone. Treatment with $10 \mu \mathrm{M}$ of troglitazone resulted in significant growth inhibition of all three cell lines (PLC/PRF/5 (B), Hep G2 (C), and HuH-7 (D)) up to 120 hours.

Troglitazone activates caspase 3 but not caspase 8

As apoptosis of cells usually involves activation of cysteine proteases of the caspase family, ${ }^{12}$ we analysed the effect of troglitazone on activation of caspase 3 and/or caspase 8 in liver cancer cells. Treatment of PLC/PRF/ 5 for 48 hours with 5 or 10 $\mu \mathrm{M}$ of troglitazone resulted in a significant increase in the activity of caspase 3, and the increase was dose dependent $(\mathrm{p}<0.01)$ (fig 5). On the other hand, the activity of caspase 8 was not influenced by treatment with troglitazone (fig 5).

Western blot analysis showed that protein expression levels of bcl-2 and bax in PLC/PRF/5 were not changed by treatment with various concentrations of troglitazone for 96 hours (fig $6)$.

\section{DISCUSSION}

It has been shown that PPAR $\gamma$ plays an important role as a regulator of lipid metabolism and a key mediator of lipid storage. Recently, the synthetic reagent troglitazone, which was originally developed as a therapeutic agent for diabetes mellitus, has been demonstrated to act as a relatively high affinity ligand for PPAR $\gamma$, and exerts growth inhibitory effects on colon cancer, breast cancer, prostate cancer, and gastric cancer. ${ }^{7-10}$ However, to our knowledge, the role of PPAR $\gamma$ in liver cancer cells has not been discussed previously. In the present

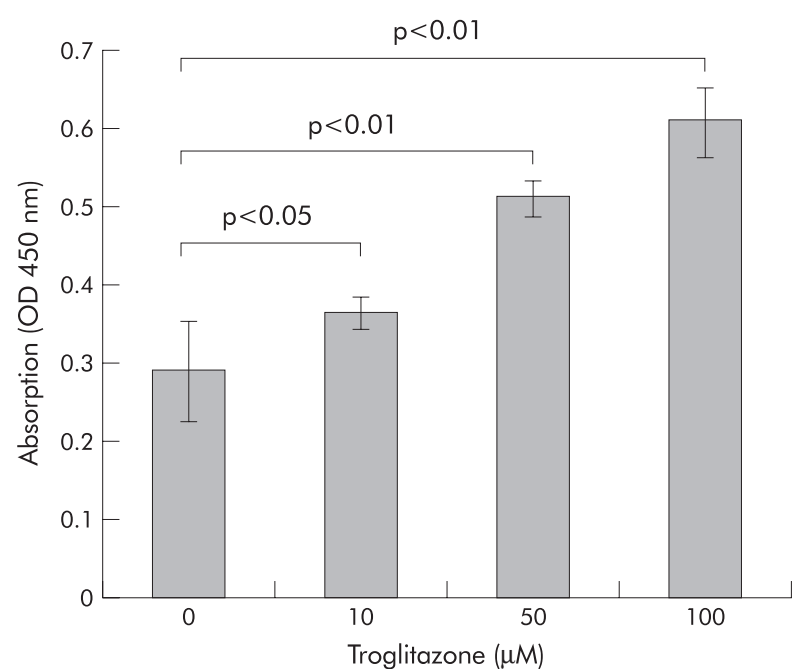

Figure 3 DNA fragmentation by ELISA assay, as measured by absorbance (OD 450 values). Culture of PLC/PRF/5 for 72 hours in the presence of troglitazone resulted in dose dependent DNA fragmentation. 

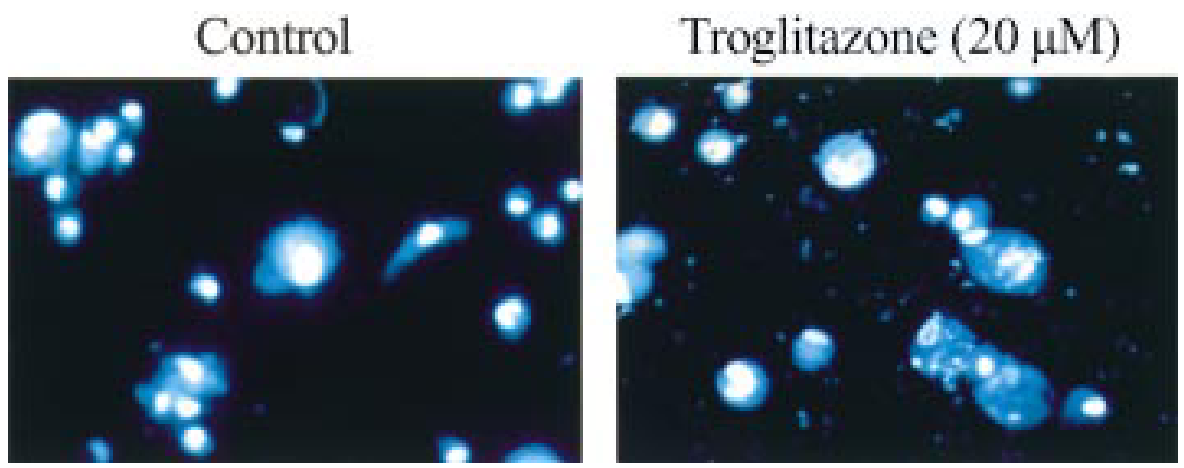

Figure 4 Detection of apoptosis of PLC/PRF/5 cells by Hoechst 33258 staining. PLC/PRF/5 treated with $20 \mu M$ of troglitazone for 72 hours showed morphological features of apoptosis. In contrast, untreated cells maintained normal chromatin patterns and cell size (original magnification $\times 200$ ).
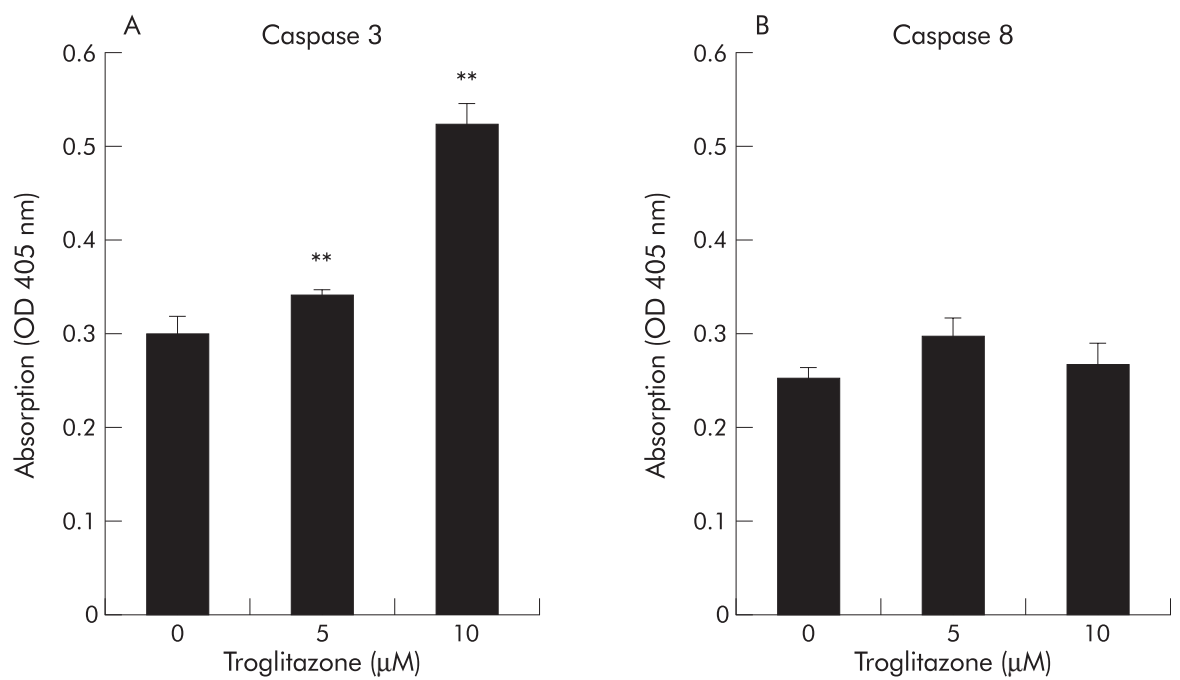

Figure 5 Effect of troglitazone on caspases 3 (A) and 8 (B) in PLC/PRF/5 cells. PLC/PRF/5 cells were treated for 48 hours with 5 or $10 \mu M$ of troglitazone. The activity of caspase 3, but not that of caspase 8, was significantly increased in response to troglitazone in a dose dependent manner. ${ }^{* *} p<0.01$

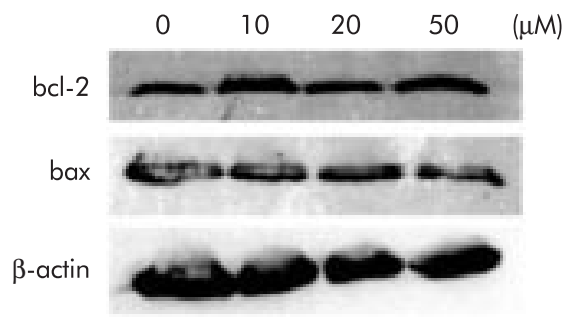

Figure 6 Effect of troglitazone on expression of bcl-2 and bax. Western blot analysis demonstrated that expression levels of bcl-2 and bax in PLC/PRF/5 were not changed by treatment with troglitazone for 96 hours.

study, we analysed the effect of troglitazone on growth and induction of apoptosis of liver cancer cells in vitro.

Our results showed that PPAR $\gamma$ mRNA was expressed at various levels in all three liver cancer cell lines. Furthermore, we also demonstrated that the growth of liver cancer cell lines was inhibited by troglitazone in a dose dependent manner. However, the rank order of the sensitivity to troglitazone did not correspond to the levels of PPAR $\gamma$ expression in these cells. HepG2, which expressed the same level of PPAR $\gamma$ mRNA as HuH-7, was relatively insensitive to the growth inhibitory effect of troglitazone. That the expression level of PPAR $\gamma$ does not parallel the growth inhibitory effect of the ligand is in agreement with the results of Elstner and colleagues ${ }^{13}$ and
Mueller and colleagues. ${ }^{8}$ Interestingly, the growth inhibitory effects of troglitazone were observed at concentrations equivalent to those detected in patients with insulin resistant diabetes mellitus treated with the same agent. This finding suggests that the clinical dose of troglitazone can potentially inhibit the growth of liver cancer cells.

To determine the underlying mechanisms of the growth inhibitory effect of troglitazone, we investigated whether troglitazone acts by inducing apoptosis of liver cancer cells. For this purpose, we performed DNA fragmentation assay and Hoechst staining. In both assays, troglitazone induced apoptosis of PLC/PRF/5 in a dose dependent manner. Chinetti and colleagues ${ }^{11}$ also reported that troglitazone induced apoptosis of human monocyte derived macrophages. However, other reports showed that apoptosis was not a major mechanism by which troglitazone induced cell growth inhibition but that differentiation of cancer cells by the ligand was the major mechanism. ${ }^{10}{ }^{13}$

We also analysed the mechanisms of troglitazone induced apoptosis. Results from our study demonstrated that troglitazone activated caspase 3 but not caspase 8 , which are cysteine proteases of the caspase family. We hypothesised that such activation of caspase 3 might indicate that apoptosis was mainly regulated by mitochondria related apoptosis factor. ${ }^{14}{ }^{15}$. However, expression levels of antiapoptotic factor bcl-2 and apoptosis inducing factor bax were not overtly affected by troglitazone treatment. In this regard, Elstner and colleagues ${ }^{13}$ 
reported that exposure of human breast cancer cells to a combination of troglitazone and all-trans-retinoic acid decreased bcl-2 protein to nearly undetectable levels.

Recent studies have examined the relationship between

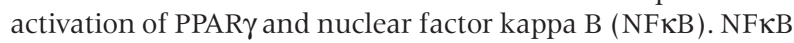
is a DNA binding protein that augments the transcription of various genes involved in cell proliferation and plays an important role in cell development, survival, and oncogenesis. ${ }^{16-18}$ In this respect, Chinetti and colleagues ${ }^{11}$ suggested that PPAR $\gamma$ induced cellular apoptosis by interfering negatively with the antiapoptotic NFKB signalling pathway. Further studies are needed to elucidate the exact mechanisms of troglitazone induced apoptosis of liver cancer cells.

Hepatocellular carcinoma is a major cause of cancer related mortality in the world and the number of new cases with this cancer is increasing. ${ }^{12}$ However, the prognosis of liver cancer is generally less favourable than other cancers. Treatment for this disease has mainly focused on surgical resection, embolisation therapy, and ethanol injection; these therapies are far from optimal. In this study, we showed that a clinical dose of troglitazone induced growth inhibition of liver cancer cells in vitro, indicating that troglitazone could be useful as a potentially effective new therapeutic agent, used alone or in combination with conventional chemotherapeutic agents, in patients with liver cancer. However, assessment of the clinical utility of this agent should await more thorough analysis of its local and systemic effects as troglitazone induced liver injury has been documented in recent studies. ${ }^{19} 20$

In summary, in the present study we have demonstrated expression of PPAR $\gamma$ in human liver cancer cells. Our results also showed that the ligand for PPAR $\gamma$, troglitazone, inhibited cell growth and induced apoptosis. The latter findings suggest that this agent might be potentially useful in the treatment of liver cancer.

\section{Authors' affiliations}

M Toyoda, H Takagi, N Horiguchi, S Kakizaki, K Sato,

H Takayama, M Mori, First Department of Internal Medicine, Gunma University School of Medicine, Maebashi, Gunma, Japan

\section{REFERENCES}

1 Omata M. Current perspectives on hepatocellular carcinoma in Oriental and African countries compared to developed western countries. Dig Dis Sci 1987;5:97-115

2 Okuda K. Hepatocellular carcinoma: recent progress. Hepatology 1992;15:948-63.

3 Warrell RP Jr, Frankel SR, Miller WH Jr, et al. Differentiation therapy of acute promyelocytic leukemia with tretinoin (all-trans-retinoic acid). $N$ Engl J Med 1991;324:1385-93.

4 Tallman MS. Differentiating therapy with all-trans retinoic acid in acute myeloid leukemia. Leukemia 1996;10(suppl 1):S12-15.

5 Mangelsdorf DJ, Thummel $C$, Beato $M$, et al. The nuclear receptor superfamily: the second decade. Cell 1995;83:835-9.

6 Spiegelman BM, Flier JS. Adipogenesis and obesity: rounding out the big picture. Cell 1996;87:377-89

7 Sarraf $P$, Mueller $E$, Jones $D$, et al. Differentiation and reversal of malignant changes in colon cancer through PPARgamma. Nat Med 1998;4:1046-52.

8 Mueller $E$, Sarraf $P$, Tontonoz $P$, et al. Terminal differentiation of human breast cancer through PPAR gamma. Mol Cell 1998;1:465-70.

9 Kubota T, Koshizuka K, Williamson EA, et al. Ligand for peroxisome proliferator-activated receptor gamma (troglitazone) has potent antitumor effect against human prostate cancer both in vitro and in vivo. Cancer Res 1998;58:3344-52.

10 Takahashi N, Okumura T, Motomura W, et al. Activation of PPARgamma inhibits cell growth and induces apoptosis in human gastric cancer cells. FEBS Lett 1999;455:135-9.

11 Chinetti G, Griglio S, Antonucci M, et al. Activation of proliferator-activated receptors alpha and gamma induces apoptosis of human monocyte-derived macrophages. J Biol Chem 1998;273:2557380.

12 Nagata S. Apoptosis by death factor. Cell 1997;88:355-65.

13 Elstner E, Muller C, Koshizuka K, et al. Ligands for peroxisome proliferator-activated receptor gamma and retinoic acid receptor inhibit growth and induce apoptosis of human breast cancer cells in vitro and in BNX mice. Proc Natl Acad Sci USA 1998;95:8806-11.

14 Liu X, Kim CN, Yang J, et al. Induction of apoptotic program in cell-free extracts: requirement for dATP and cytochrome c. Cell 1996:86:147-57.

15 Zou H, Henzel WJ, Liu X, et al. Apaf-1, a human protein homologous to C. elegans CED-4, participates in cytochrome c-dependent activation of caspase-3. Cell 1997;90:405-13.

16 Beg AA, Sha WC, Bronson RT, et al. Embryonic lethality and liver degeneration in mice lacking the RelA component of NF-kappa B. Nature 1995;376:167-70.

17 Baldwin AS Jr. The NF-kappa B and I kappa B proteins: new discoveries and insights. Annu Rev Immunol 1996;14:649-83.

18 Baeverle PA, Baltimore D. NF-kappa B: ten years after. Cell 1996;87:13-20

19 Watkins PB, Whitcomb RW. Hepatic dysfunction associated with troglitazone. N Engl J Med 1998;338:916-17.

20 Iwase $M$, Yamaguchi M, Yoshinari M et al. A Japanese case of liver dysfunction after 19 months of troglitazone treatment. Diabetes Care 1999;22:1382-4. 